\title{
Prevalencia de Fasciola hepatica en Bovinos Sacrificados en la Planta de Beneficio del Municipio de Une, Cundinamarca, Colombia
}

\author{
Prevalence of Fasciola hepatica in Cattle in the Slaughterhouse of the \\ Municipality of Une, Cundinamarca, Colombia \\ Julio César Giraldo Forero ${ }^{1,3}$, Adriana María Díaz Anaya ${ }^{2,4}$, \\ Martín Orlando Pulido Medellín ${ }^{2,5}$
}

\section{Resumen}

El objetivo del presente estudio fue determinar la prevalencia de Fasciola hepatica en bovinos sacrificados en la planta de beneficio del municipio de Une, Cundinamarca, Colombia. El tamaño muestral fue calculado con base a la tasa de sacrificio de 2013 con $6 \%$ de incremento $(n=142)$. Durante el eviscerado se recolectaron distomas adultos del ducto biliar, así como muestras del contenido biliar y materia fecal. En el examen coproparasitológico se empleó la técnica de Ritchie-Frick modificada. La prevalencia de $F$. hepatica fue $39.4 \%$ por presencia de huevos en el contenido biliar, $32.4 \%$ por presencia de parásitos adultos en los ductos biliares y $15.5 \%$ por presencia de huevos en materia fecal. El valor porcentual de animales positivos por presencia de estadio adulto, así como huevos en bilis y materia fecal, fue de $15.5 \%$.

Palabras clave: Fasciola hepatica, estudio transversal, bovinos, trematodo, bilis

\section{Abstract}

The aim if this study was to determine the prevalence of Fasciola hepatica in cattle at the slaughterhouse of the municipality of Une, Cundinamarca, Colombia. Sample size was calculated based on the slaughter rate of 2013 plus $6 \%$ increase $(n=142)$. Adult parasites were collected from the biliary duct. Bile and faecal material were also collected.

\footnotetext{
${ }^{1}$ Grupo de Investigación en Parasitología y Microbiología Tropical - GIPAMT, Programa de Biología, Facultad de Ingenierías, Administración y Ciencias Básicas, Universidad INCCA de Colombia, Bogotá, Colombia

${ }^{2}$ Grupo de Investigación en Medicina Veterinaria y Zootecnia - GIDIMEVETZ, Universidad Pedagógica y Tecnológica de Colombia, Tunja, Colombia

${ }^{3}$ E-mail: jcesargiraldo@gmail.com

${ }^{4}$ E-mail:adrima43@gmail.com

${ }^{5}$ E-mail:mopm1@hotmail.com
}

Recibido: 17 de abril de 2016

Aceptado para publicación: 11 de agosto de 2016 
The modified Ritchie-Frick technique was used to analyse faecal samples. The prevalence of F. hepatica was $39.4 \%$ based on eggs in the bile, $32.4 \%$ based on the presence of adult parasites in biliary ducts and $15.5 \%$ based on the presence of eggs in faecal samples. Positive animals for presence of adult parasites plus eggs in bile and faecal samples was $15.5 \%$.

Key words: Fasciola hepatica, cross-sectional study, cattle, trematode, bile

\section{INTRODUCCIÓN}

La fasciolosis, causada por Fasciola hepatica, es una parasitosis de importancia ganadera a escala mundial, y que puede transmitirse al hombre, por lo que es considerada la zoonosis ocasionada por helmintos de mayor prevalencia en el mundo (Haridy et al., 2002). Este trematodo se aloja en el conducto hepático y vías biliares de humanos y rumiantes. La infección humana constituye un serio problema de salud pública, afectando cerca de 2.4 millones de personas a nivel mundial (Dietrich et al., 2015). Su ciclo de vida involucra a caracoles del género Lymnaea como huésped intermediario, donde el miracidio pasa por varios estadios hasta llegar a cercaria, la cual es liberada del caracol formando quistes conocidos como metacercarias y que constituyen las formas infectantes. Estos quistes se adhieren a la vegetación acuática, la cual es ingerida por mamíferos o personas, adquiriendo de esa forma la infección (Castro y Becerra, 2011; Lazo et al., 2013).

Los bovinos y los ovinos son los principales hospedadores definitivos, pero puede encontrarse en una gran variedad de animales como cabras, burros, búfalos, caballos, camellos y conejos (Farag, 1998; Kalu et al., 2015). La fasciolosis causa, además, disminución de la producción de leche y carne y el decomiso de los hígados infectados (González et al., 2013). Si la enfermedad se manifiesta clínicamente, se observa debilidad, anorexia, palidez y edema de mucosa y conjuntiva, y dolor a la palpación en la zona de proyección hepática. La infección de rumiantes domésticos con F. hepatica y F. gigantica causa pérdidas económicas estimadas en más de US\$ 2000 millones por año en el sector agrícola mundial con más de 600 millones de animales infectados (Recalde et al., 2014), mientras que las pérdidas en Colombia son de 12483 millones de pesos por año (Becerra, 2001).

Las plantas de beneficio ofrecen una excelente oportunidad para detectar enfermedades, tanto de importancia económica como en la salud pública. Los hígados infectados por este parásito son regularmente decomisados tras la inspección en las plantas de beneficio, repercutiendo en pérdidas económicas a los ganaderos. Por otro lado, información obtenida en las plantas de beneficio puede contribuir en la comprensión y la epidemiología de las enfermedades (OladeleBukola y Odetokun, 2014).

La mayor prevalencia de fascioliosis bovina en Colombia se presenta en las zonas ganaderas de climas fríos, donde es endémica y afecta al $25 \%$ del ganado lechero (Estrada et al., 2006). Cundinamarca cuenta las reseñas más antiguas y numerosas del país sobre fasciolosis bovina y humana; sin embargo, gran parte del departamento está por investigar. La temperatura necesaria para que la $F$. hepatica pueda desarrollarse en el caracol es superior a $10{ }^{\circ} \mathrm{C}$; posiblemente por esto, el municipio de Une, en el departamento de Cundinamarca, reúne las condiciones ecológicas para favorecer el establecimiento del parásito, pero donde no se tiene registro oficial de la zoonosis. Por lo anterior, esta 
investigación se realizó con el fin de establecer la prevalencia de fasciolosis en los bovinos sacrificados en la planta de beneficio del municipio de Une, Cundinamarca.

\section{Materiales y Métodos}

El municipio de Une se encuentra ubicado en la Región de Oriente, al sur del departamento de Cundinamarca, Colombia. Posee una extensión de $233360 \mathrm{~km}^{2}$ y se encuentra a una altura de $2376 \mathrm{~m}$. La temperatura media anual de la zona es de $16^{\circ} \mathrm{C}$.

En el muestreo se consideraron los bovinos aptos para sacrificio en la planta de beneficio del municipio de Une, con registro de control sanitario expedido por la Unidad Municipal de Asistencia Técnica Agropecuaria (UMATA), y que podían proceder del municipio o ser traídos de otros municipios. Teniendo en cuenta el plan de desarrollo de la alcaldía municipal, donde está consignada la tasa de sacrificio del año 2013, se estableció un tamaño muestral de 134 bovinos, con $6 \%$ de incremento por pérdidas posibles, para un total de 142 muestras (Recalde et al., 2014). Los animales en su mayoría pertenecían a producciones de tipo doble propósito con manejo semiintensivo.

El rango de edades de los 142 bovinos evaluados estuvo representado de la siguiente manera: $<2$ años: $24.6 \%$, 2-4 años: $65.5 \%$, 5-8 años: $7.8 \%$; y $>8$ años: $2.1 \%$.

La toma y procesamiento de las muestras se llevó a cabo en los meses de enero a agosto de 2014, a partir de una visita semanal a la planta de beneficio durante el día de mayor sacrificio. Se registró la procedencia de los animales. Se colectó por cada animal una muestra de $50 \mathrm{ml}$ de bilis y $30 \mathrm{~g}$ de materia fecal; además, se realizaron observaciones macroscópicas del estado de los hígados y se recolectaron parásitos adultos por disección del ducto hepático en el momento del eviscerado. Los parásitos adultos fueron llevados al laboratorio para ser lavados con solución salina $(\mathrm{NaCl} 0.9 \%)$, donde una parte fueron preservados en buffer fosfato salino con antibiótico (cloranfenicol $250 \mathrm{mg} /$ $500 \mathrm{ml}$ ) para producción de antígeno, otra parte fue conservada en alcohol etílico al $70 \%$ para estudios moleculares posteriores, $\mathrm{y}$ otra fue preservada en formol al 10\% y destinada al banco de parásitos del laboratorio.

Las muestras de material fecal fueron almacenadas, previa adición de conservantes como el reactivo de MIF (merthiolate-yodoformol). Todas las muestras se mantuvieron refrigeradas y fueron trasladadas al laboratorio.

Las muestras de heces se procesaron por la técnica de Ritchie-Frick modificada. Para esto, las muestras fueron disueltas en solución salina, filtradas en doble gasa y centrifugadas a $450 \mathrm{~g}$ durante $5 \mathrm{~min}$; luego se eliminó el sobrenadante y el precipitado se mezcló con $10 \mathrm{ml}$ de formol al $10 \%$ y $2 \mathrm{ml}$ de bencina de petróleo, donde fue centrifugado por segunda vez. Después de eliminar el sobrenadante, el precipitado se almacenó en tubos Eppendorf para su análisis posterior.

El contenido biliar fue centrifugado a $450 \mathrm{~g}$ durante $10 \mathrm{~min}$, con el fin de aislar los huevos en el sedimento resultante. La lectura de los sedimentos resultantes se realizó por triplicado y con doble ciego, montando el concentrado de bilis y materia fecal sobre un portaobjetos con solución de lugol. Se observaron al microscopio con objetivos de 10X y 40X (América y Banchero, 2013).

El estudio realizado fue de tipo descriptivo, de corte transversal, con toma única de muestra. La información obtenida fue analizada empleando el software EPI INFO 7.1.3.

\section{Resultados}

En el 39.4\% (56/142) de las muestras de contenido biliar y en $15.5 \%$ de las muestras fecales se demostró la presencia de hue- 
Cuadro 1. Frecuencia de huevos y parásitos adultos de Fasciola hepatica en hígados de bovinos sacrificados en la planta de beneficio del municipio de Une, Cundinamarca (2014), según localidad de procedencia

\begin{tabular}{|c|c|c|c|c|}
\hline Localidad & $\begin{array}{c}\text { Muestras } \\
\text { (n) }\end{array}$ & $\begin{array}{l}\text { Presencia de } \\
\text { huevos en bilis } \\
(\%)\end{array}$ & $\begin{array}{l}\text { Presencia de } \\
\text { huevos en } \\
\text { materia fecal } \\
(\%)\end{array}$ & $\begin{array}{c}\text { Presencia de } \\
\text { parásito adultos } \\
(\%)\end{array}$ \\
\hline Alagunas & 2 & 3.6 & 0 & 3.6 \\
\hline Alto mesa & 1 & 1.8 & 1.8 & 1.8 \\
\hline Bolsitas & 2 & 3.6 & 0 & 3.6 \\
\hline Chamizal & 1 & 1.8 & 0 & 0 \\
\hline Chipa que $^{1}$ & 8 & 14.3 & 5.4 & 14.2 \\
\hline Combura & 1 & 1.8 & 1.8 & 1.8 \\
\hline El Ramal & 1 & 1.8 & 1.8 & 1.8 \\
\hline La Hoja & 3 & 5.4 & 1.8 & 3.6 \\
\hline La Mesa & 6 & 10.7 & 5.4 & 10.7 \\
\hline La Vega $^{1}$ & 2 & 3.6 & 1.8 & 3.6 \\
\hline Puente T & 1 & 1.8 & 0 & 0 \\
\hline Queca & 1 & 1.8 & 0 & 1.8 \\
\hline Raspagos & 3 & 5.4 & 1.8 & 5.4 \\
\hline Rincón Gr. & 1 & 1.8 & 1.8 & 1.8 \\
\hline Salitre & 7 & 12.5 & 3.6 & 1.8 \\
\hline San Luis & 12 & 21,4 & 10.6 & 21.4 \\
\hline San Pablo & 1 & 1.8 & 0 & 1.8 \\
\hline Santuario & 2 & 3.6 & 1.8 & 3.6 \\
\hline Trilladora & 1 & 1.8 & 0 & 0 \\
\hline Total & 56 & 100 & 39.4 & 82.3 \\
\hline
\end{tabular}

${ }^{1}$ Localidades fuera del municipio de Une

vos de F. hepatica, en tanto que en el $32.4 \%$ de los hígados se encontró parásitos adultos en los ductos biliares. Asimismo, en el examen macroscópico de los hígados se encontró que el $35.9 \%$ presentaba el moteado característico de cirrosis causada por $F$. hepatica.

El 56.3\% (80/142) de los hígados fueron de bovinos machos, de los cuales el 35\% $(28 / 80)$ fue positivo para el digeneo. Asimis- mo, el 43.7\% (62/142) correspondió a hembras donde el 45\% (28/62) presentó el parásito; sin que hubiera diferencia estadística entre sexos. La frecuencia de hígados de animales parasitados según procedencia se muestra en el Cuadro 1.

En relación con el grupo racial, los bovinos de raza Normanda aportaron el 56.3\% (80/142) de los animales, donde el 52.5\% (42/80) se encontró parasitado. Asimismo, los 
bovinos criollos y Brahman correspondieron al 25.3 y $16.9 \%$ de la población, donde el 33.3 y $0 \%$, respectivamente estuvieron parasitados. Otras razas y cruces representaron el resto de la población muestreada.

\section{Discusión}

La prevalencia general hallada fue del $39.4 \%$ cuando el resultado de prevalencia esperado era de $25 \pm 15 \%$, según el reporte nacional para este agente etiológico en bovinos (Pulido et al., 2011). Otros estudios nacionales reportan $3.7 \%$ de prevalencia en el departamento del Quindio (Recalde et al., 2014) y más de $30 \%$ en la región lechera altoandina de Antioquia (López et al., 2008). Asimismo, en dos hatos lecheros del norte y del oriente de Antioquia se reportaron prevalencias entre 10.5 y $94.6 \%$ para $F$. hepatica por coprología Dennis y de 49.5 y $93.1 \%$ por ELISA (Bedoya et al., 2007). Por otro lado, prevalencias diversas han sido reportadas en otros países (Kithuka et al., 2002; Ezatpour et al., 2014; Magaji et al., 2014; Oladele-Bukola y Odetokun, 2014), las cuales son dependientes del medio ambiente, sistemas de manejo, raza y técnicas de laboratorio.

Los tres tipos de muestras empleadas para el diagnóstico post mortem de $F$. hepatica demuestran que el parásito no presenta una ovoposición constante. Como se pudo observar, la presencia del adulto no garantiza el hallazgo de huevos en bilis o en materia fecal (Chirinos et al., 2000; Martínez et al., 2013). Por otro lado, destaca la presencia de huevos en bilis al aportar la frecuencia de casos positivos más elevada y el análisis copro-parasitológico por ser el menos sensible (Ticona et al., 2010). Resultados similares fueron encontrados por Rapsch et al. (2006), donde los huevos en bilis y la técnica de ELISA aportaron la mayor sensibilidad (93.4 y 91.7\%, respectivamente), mientras que las técnicas coprológicas e ins- pección de los hígados arrojaron una prevalencia de 69 y $63.2 \%$, respectivamente. La frecuencia de huevos en bilis fue mayor que los parásitos encontrados en la inspección post mortem, dado que en ocasiones es difícil encontrar los adultos en los ductos hepáticos debido a la fibrosis presente o por el encapsulamiento de los parásitos.

Cabe resaltar que la visualización del parásito adulto por disección del ducto biliar, presencia de huevos en contenido biliar y materia fecal, así como la observación de hígados con signos sugestivos de cirrosis hepática («pigmentación de sal y pimienta») se observó en el $21.4 \%(12 / 56)$ de los casos. Esto indica claramente que no siempre se va a cumplir con estos cuatro parámetros como una constante para esta entidad parasitaria (Espinoza et al., 2010; Swarnakar y Sanger, 2014).

Individuos de la raza Normanda fueron los más frecuentes en la planta de beneficio y fueron además los que presentaron el mayor valor porcentual de $F$. hepatica (42/80, $52.5 \%$ ). Esta raza es la más abundante en la zona, posiblemente porque se adapta fácilmente a los climas fríos y de páramo, donde se hallan las condiciones ambientales que propician el desarrollo de los caracoles Lymnaea que actúan como hospederos intermediarios de F. hepatica (Nguyen et al., 2011). Los bovinos que se encuentran al pastoreo y que beben agua proveniente de aguas corrientes están expuestos a factores que predisponen a la infección por $F$. hepatica (González et al., 2013; Zumaquero et al., 2013).

La mayor prevalencia de $F$. hepatica registrada en este estudio (39.4\%) con relación al promedio nacional indica que probablemente este último valor se halle subestimado o que el área en estudio corresponda a una zona hiperendémica (Wilches et al., 2009; Naquira, 2010; Pinedo et al., 2010), lo que permite suponer que esta zoonosis genera riesgo de infección para la población humana en la región. 


\section{Agradecimiento}

Los autores agradecen a la Alcaldía Municipal de Une, Cundinamarca, a la Unidad Municipal de Asistencia Técnica Agropecuaria (UMATA) y a la administración de la planta de beneficio. Asimismo, a la Biól. Luz Elena Velásquez, del laboratorio de Malacología Médica y Trematodos del Programa de Estudio y Control de Enfermedades Tropicales (PECET) de la Universidad de Antioquia.

\section{Literatura Citada}

1. América M, Banchero G. 2013. Parasitosis gastrointestinales de ovinos y bovinos: situación actual y avances de la investigación. Revista INIA 34: 10-15.

2. Bedoya J, Gómez V, Pérez, R, Calle J, Velásquez L. 2007. Evaluación de un test de ELISA para el diagnóstico de la fasciolosis bovina en condiciones de campo e identificación del molusco hospedador. Rev Col Cienc Pec 20: 596 (Resumen).

3. Becerra M. 2001. Consideraciones sobre estrategias sostenibles para el control de Fasciola hepatica en Latinoamérica. Rev Col Cienc Pec 14: 28-35.

4. Castro N, Becerra W. 2011. Foco de fasciolisis ovina en hacienda en la vereda Presidente, municipio de Chitagá, Norte de Santander, Colombia. Bistua 9(2): 64-72.

5. Chirinos A, Chirinos N, Román $R$, Homez G, Pirela H, Rodríguez N. 2000. Distomatosis hepática bovina a nivel de dos mataderos industriales del estado Zulia, Venezuela. Rev Científ. FVC-LUZ 10: 297-302.

6. Dietrich C, Kabaalioglu A, Brunetti E, Richter J. 2015. Fasciolosis. Z Gastroenterol 53: 285-290. doi: 10.1055/ s-0034-1385728

7. Espinoza J, Terashima A, Herrera P, Marcos L. 2010. Fasciolosis humana y animal en el Perú: impacto en la econo- mía de las zonas endémicas. Rev Perú Med Exp Salud Pública 27: 604-612. doi: 10.1590/S1726-46342010000400018

8. Estrada V, Gómez M, Velásquez, L. 2006. La higiene del ganado y la fasciolosis bovina, Medellín y Rionegro, 1914-1970. IATREIA 19: 393-407.

9. Ezatpour B, Hasanvand A, Azami A, Mahmoudvand H, Anbari K. 2014. A slaughterhouse study on prevalence of some helminths of cattle in Lorestan provience, west Iran. Asian Pacific J Trop Dis 4: 416-420. doi: 10.1016/S22221808(14)60599-5

10. Farag HF. 1998. Human fascioliasis in some countries of the Eastern Mediterranean Region. East Mediterr Health J 4: 156-160.

11. González C, Sánchez G, Castro C, Carmona C, Molina F, Velásquez $L$. 2013. Control de Fasciola hepatica en el agua de consumo animal a través de filtración rápida y lenta. Rev EIA Esc Ing Antioq 10: 133-141.

12. Haridy F, Morsy T, Gawish N, Antonios T, Abdel Gawad A. 2002. The potential reservoir role of donkeys and horses in zoonotic fascioliasis in Gharbia Governorate, Egypt. J Egypt Soc Parasitol 32: 561-570.

13. Kalu E, Akpabio U, Gloria D. 2015. A case of chronic fascioliasis in a cattle slaughtered at Ubakala abattoir. J Vet Adv 5: 1017-1022. doi: 10.5455/ jva.20150628020845

14. Kithuka J, Maingi N, Njeruh F, Ombui J. 2002. The prevalence and economic importance of bovine fasciolosis in Kenya - an analysis of abattoir data. Onderstepoort J Vet Res 69: 255-262.

15. Lazo L, Garrido $R$, Cárdenas B, Torreblanca J. 2013. Extracción endoscópica por CPRE de Fasciola hepatica viva: reporte de dos casos y revisión de la literatura. Rev Gatroenterol Perú 33: 75-81.

16. López, L, Romero J, Velásquez L. 2008. Aislamiento de Paramphistomidae en vacas de leche y en el hospedador inter- 
mediario (Lymnaea truncatula y Lymnaea columella) en una granja del trópico alto en el occidente de Colombia. Rev Colomb Cienc Pec 21: 9-18.

17. Magaji A, Ibrahim K, Salihu M, Saulawa M, Mohammed A, Musawa A. 2014. Prevalence of fascioliasis in cattle slaughtered in Sokoto Metropolitan abattoir, Sokoto, Nigeria. Hindawi Publishing Corporation Adv Epidemiol Article ID 247258. doi: 10.1155/2014/ 247258

18. Martínez M, Robles D, Martínez J, Cordero C, Famularo R, Fernández $N$, González-C, et al. 2013. Prevalence of gastrointestinal nematodes and Fasciola hepatica in sheep in the northwest of Spain: relation to climatic conditions and/or man-made environmental modifications. Parasit Vectors 6: 282. doi: 10.1186/1756-33056-282

19. Naquira C. 2010. Las zoonosis parasitarias: problema de salud pública en el Perú. Rev Peru Med Exp Salud Publica 27: 494-97. doi: 10.1590/S172646342010000400001

20. Nguyen TG, Le TH, Dao TH, Tran TL, Praet N, Speybroeck N, Vercruysse J, Dorny P. 2011. Bovine fasciolosis in the human fasciolosis hyperendemic Binh Dinh province in Central Vietnam. Acta Trop 117: 19-22. doi:10.1016/ j.actatropica.2010.09.003

21. Oladele-Bukola M, Odetokun I. 2014. Prevalence of bovine fasciolosis at the Ibadan municipal abattoir, Nigeria. African J Food Agric Nutrit Deve 14: 9055-9070.

22. Pinedo V, Chávez V, Casas A, Suárez A, Sánchez P, Huamán U. 2010. Prevalencia de trematodes de la familia Paramphistomatidae en bovinos del distrito de Yurimaguas, provincia de alto Amazonas, Loreto. Rev Inv Vet Perú 21: 161-167. doi: 10.15381/rivep.v21i2.132
23. Pulido Villamarín A, Castañeda R, Arbelaez G 2011. Fasciola hepatica: Pedagogía de diagnóstico por laboratorio y su situación en Colombia. REDVET 12(5B). http://www.veterinaria.org/revistas/redvet/n050511B/051124.pdf

24. Rapsch C, Schweizer G, Grimm F, Kohler L, Bauer C, Deplazes P, Braun $\boldsymbol{U}$, Torgerson P. 2006. Estimating the true prevalence of Fasciola hepatica in cattle slaughtered in Switzerland in the absence of an absolute diagnostic test. Int J Parasitol 36: 1153-1158. doi: 10.1016/j.ijpara.2006.06.001

25. Recalde-Reyes D, Padilla L, Giraldo M, Toro L, González M, Castaño J. 2014. Prevalencia de Fasciola hepatica en humanos y bovinos en el departamento del Quindío-Colombia 2012-2013. Infectio 18: 153-157. doi: 10.1016/j.infect.2014.09.001

26. Swarnakar G, Sanger B. 2014. Epidemiological study of liver fluke (Trematoda: Digenea) in domestic ruminants of Udaipur district. Int J Curr Microbiol App Sci 3: 632-640.

27. Ticona D, Chávez A, Casas G, Chavera A, Li O. 2010. Prevalencia de Fasciola hepatica en bovinos y ovinos de Vilcashuamán, Ayacucho. Rev Inv Vet Perú 21: 168-174.

28. Wilches C, Jaramillo J, Muñoz, D, Robledo M, Vélez I. 2009. Presencia de infestación por Fasciola hepatica en habitantes del valle de San Nicolás, oriente antioqueño. Infectio 13: 92-99. doi: 10.1016/S0123-9392(09)70730-1

29. Zumaquero J, Sarracent J, Rojas R, Rojas L, Martínez, Y, Valero M, Mas S. 2013. Fascioliasis and intestinal parasitoses affecting school children in Atlixco, Puebla State, México: epidemiology and treatment with nitazoxanide. PLOS Negl Trop Dis 7: e2553. doi: 10.1371/journal.pntd.0002553 